\title{
An Alternative Proof of the Largest Number of Maximal Independent Sets in Connected Graphs Having at Most Two Cycles
}

\author{
Min-Jen Jou', Jenq-Jong Lin² \\ ${ }^{1}$ Department of Information Technology, Ling Tung University, Taiwan \\ ${ }^{2}$ Department of Finance, Ling Tung University, Taiwan \\ Email:mjjou@teamail.ltu.edu.tw,jjlin@teamail.ltu.edu.tw
}

How to cite this paper: Jou, M.-J. and Lin, J.-J. (2016) An Alternative Proof of the Largest Number of Maximal Independent Sets in Connected Graphs Having at Most Two Cycles. Open Journal of Discrete Mathematics, 6, 227-237.

http://dx.doi.org/10.4236/ojdm.2016.64019

Received: June 28, 2016

Accepted: August 22, 2016

Published: August 25, 2016

Copyright $\odot 2016$ by authors and Scientific Research Publishing Inc. This work is licensed under the Creative Commons Attribution International License (CC BY 4.0). http://creativecommons.org/licenses/by/4.0/ (c) (i) Open Access

\begin{abstract}
G. C. Ying, Y. Y. Meng, B. E. Sagan, and V. R. Vatter [1] found the maximum number of maximal independent sets in connected graphs which contain at most two cycles. In this paper, we give an alternative proof to determine the largest number of maximal independent sets among all connected graphs of order $n \geq 12$, which contain at most two cycles. We also characterize the extremal graph achieving this maximum value.
\end{abstract}

\section{Keywords}

Maximal Independent Set, Connected Graph Having at Most Two Cycles

\section{Introduction}

Let $G=(V, E)$ be a simple undirected graph. An independent set is a subset $S$ of $V$ such that no two vertices in $S$ are adjacent. A maximal independent set is an independent set that is not a proper subset of any other independent set. The set of all maximal independent sets of a graph $G$ is denoted by $M I(G)$ and its cardinality by $\operatorname{mi}(G)$.

The problem of determining the largest value of $\mathrm{mi}(G)$ in a general graph of order $n$ and those graphs achieving the largest number was proposed by Erdös and Moser, and solved by Moon and Moser [2]. It was then extensively studied for various classes of graphs in the literature, including trees, forests, (connected) graphs with at most one cycle, bipartite graphs, connected graphs, $k$-connected graphs, (connected) triangle-free graphs; for a survey see [3]. Recently, Jin and Li [4] determined the second largest number of maximal independent sets among all graphs of order $n$. 
There are results on independent sets in graphs from a different point of view. The Fibonacci number of a graph is the number of independent vertex subsets. The concept of the Fibonacci number of a graph was introduced in [5] and discussed in several papers [6] [7]. In addition, Jou and Chang [8] showed a linear-time algorithm for counting the number of maximal independent sets in a tree.

Jou and Chang [9] determined the largest number of maximal independent sets among all graphs and connected graphs of order $n$, which contain at most one cycle. Later B. E. Sagan and V. R. Vatter [10] found the largest number of maximal independent sets among all graphs of order $\mathrm{n}$, which contain at most $r$ cycles. In 2012, Jou [11] settled the second largest number of maximal independent sets in graphs with at most one cycle. G. C. Ying, Y. Y. Meng, B. E. Sagan, and V. R. Vatter [1] found the maximum number of maximal independent sets in connected graphs which contain at most two cycles. In this paper, we give an alternative proof to determine the largest number of maximal independent sets among all connected graphs of order $n \geq 12$, which contain at most two cycles. We also characterize the extremal graph achieving this maximum value.

For a graph $G=(V, E)$, the cardinality of $V(G)$ is called the order, and it is denoted by $|G|$. The neighborhood $N(x)$ of a vertex $x \in V(G)$ is the set of vertices adjacent to $x$ in $G$ and the closed neighborhood $N[x]$ is $\{x\} \cup N(x)$. The degree of $x$ is the cardinality of $N(x)$, and it is denoted by $\operatorname{deg}(x)$. A vertex $x$ is said to be a leaf if $\operatorname{deg}(x)=1$. For a set $A \subseteq V(G)$, the deletion of $A$ from $G$ is the graph $G-A$ obtained from $G$ by removing all vertices in $A$ and their incident edges. Two graphs $G_{1}$ and $G_{2}$ are disjoint if $V\left(G_{1}\right) \cap V\left(G_{2}\right)=\varnothing$. The union of two disjoint graphs $G_{1}$ and $G_{2}$ is the graph $G_{1} \cup G_{2}$ with vertex set $V\left(G_{1} \cup G_{2}\right)=V\left(G_{1}\right) \cup V\left(G_{2}\right)$ and edge set $E\left(G_{1} \cup G_{2}\right)=E\left(G_{1}\right) \cup E\left(G_{2}\right)$. If a graph $G$ is isomorphic to another graph $H$, we denote $G=H$. Denote $K_{n}$ a complete graph of order $n$ and $C_{n}$ a cycle of order n. The join of two disjoint graphs $G_{1}$ and $G_{2}$ is the graph $G_{1}+G_{2}$ with vertex set $V\left(G_{1} \cup G_{2}\right)=V\left(G_{1}\right) \cup V\left(G_{2}\right)$ and edge set $E\left(G_{1} \cup G_{2}\right)=E\left(G_{1}\right) \cup E\left(G_{2}\right) \cup\left\{u v: u \in V\left(G_{1}\right)\right.$ and $\left.v \in V\left(G_{2}\right)\right\}$. The star-product of two disjoint graphs $G_{1}$ and $G_{2}$ is the graph $G_{1} \times G_{2}$ with vertex set $V\left(G_{1} \cup G_{2}\right)=V\left(G_{1}\right) \cup V\left(G_{2}\right)$ and edge set $E\left(G_{1} \cup G_{2}\right)=E\left(G_{1}\right) \cup E\left(G_{2}\right) \cup\left\{v_{1} v_{2}\right\}$, where $v_{i}$ is a vertex with maximum degree in $G_{i}$ for $i=1,2$.

\section{Preliminary}

The following results are needed.

Lemma 1. ([12] [13]) For any vertex $x$ in a graph $G$, the following hold.

1) $\operatorname{mi}(G) \leq m i(G-x)+m i\left(G-N_{G}[x]\right)$.

2) If $x$ is a leaf adjacent to $y$, then $\operatorname{mi}(G)=m i\left(G-N_{G}[x]\right)+m i\left(G-N_{G}[y]\right)$.

Lemma 2. ([13]) If $G$ is the union of two disjoint graphs $G_{1}$ and $G_{2}$, then $\operatorname{mi}(G)=\operatorname{mi}\left(G_{1}\right) m i\left(G_{2}\right)$.

Lemma 3. Let $a, b, n$ be integers such that $0<a<\frac{n}{2}<b$ and let $f(x)=2^{x}+2^{n-x}$.

Then $f(x) \leq \max \{f(a), f(b)\}$. 
Proof. The derivative of $f(x)$ is

$$
f^{\prime}(x)=2^{x}(\ln 2)-\left(2^{n-x}\right)(\ln 2)=(\ln 2)\left[2^{x}-2^{n-x}\right] .
$$

So $f^{\prime}(x)<0$ for any $x \in\left(a, \frac{n}{2}\right)$ and $f^{\prime}(x)>0$ for any $x \in\left(\frac{n}{2}, b\right)$. Then $f(x)$ is decreasing on $\left(a, \frac{n}{2}\right)$ and $f(x)$ is increasing on $\left(\frac{n}{2}, b\right)$. Hence $f(x) \leq \max \{f(a), f(b)\}$.

Theorem 1. ([9]) If $T$ is a tree with $n \geq 1$ vertices, then $\operatorname{mi}(G) \leq t(n)$, where

$$
t(n)= \begin{cases}2^{\frac{n-1}{2}}, & \text { if } n \text { is odd; } \\ 2^{\frac{n-2}{2}}+1, & \text { if } n \text { is even. }\end{cases}
$$

Furthermore, $\operatorname{mi}(T)=t(n)$ if and only if $T \in T(n)$, where

$$
T(n)= \begin{cases}B\left(1, \frac{n-1}{2}\right), & \text { if } n \text { is odd; } \\ \left.B\left(2, \frac{n-2}{2}\right)\right) \text { or } B\left(4, \frac{n-4}{2}\right), & \text { if } n \text { is even, }\end{cases}
$$

where $B(i, j)$ is the set of batons, which are the graphs obtained from a basic path $P$ of $i \geq 1$ vertices by attaching $j \geq 0$ paths of length two to the endpoints of $P$ in all possible ways (see Figure 1).

Theorem 2. ([9]) If $F$ is a forest with $n \geq 1$ vertices, then $\operatorname{mi}(G) \leq f(n)$, where

$$
f(n)= \begin{cases}2^{\frac{n-1}{2}}, & \text { if } n \text { is odd } ; \\ 2^{\frac{n}{2}}, & \text { if } n \text { is even. }\end{cases}
$$

Furthermore, $\operatorname{mi}(F)=f(n)$ if and only if $F \in F(n)$, where

$$
F(n)= \begin{cases}B\left(1, \frac{n-1-2 s}{2}\right) \cup s P_{2}, & \text { if } n \text { is odd ; } \\ \frac{n}{2} P_{2}, & \text { if } n \text { is even, }\end{cases}
$$

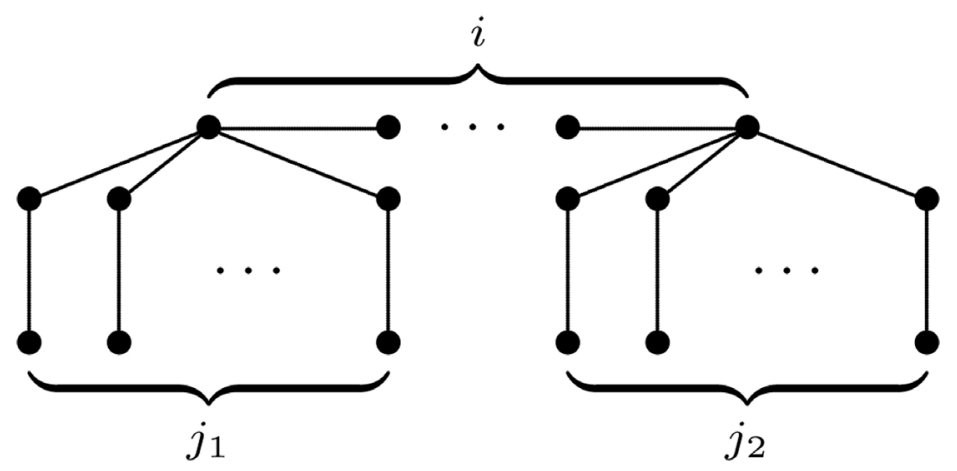

Figure 1 . The baton $B(i, j)$ with $j=j_{1}+j_{2}$. 
where $0 \leq s \leq \frac{n-1}{2}$.

Theorem 3. ([9]) If $G$ is a graph of order $n \geq 2$ vertices with at most one cycle, then $\operatorname{mi}(G) \leq g(n, 1)$, where

$$
g(n, 1)= \begin{cases}2^{\frac{n}{2}}, & \text { if } n \text { is even; } \\ 3 \cdot 2^{\frac{n-3}{2}}, & \text { if } n \text { is odd. }\end{cases}
$$

Furthermore, $\operatorname{mi}(G)=g(n, 1)$ if and only if $G=G(n, 1)$, where

$$
G(n, 1)= \begin{cases}\frac{n}{2} K_{2}, & \text { if } n \text { is even; } \\ K_{3} \cup \frac{n-3}{2} K_{2}, & \text { if } n \text { is odd. }\end{cases}
$$

Theorem 4. ([11]) If $G$ is a graph of order $n \geq 4$ with at most one cycle such that $G \neq G(n, 1)$, then $\operatorname{mi}(G) \leq g^{\prime}(n, 1)$, where

$$
g^{\prime}(n, 1)= \begin{cases}3 \cdot 2^{\frac{n-4}{2}}, & \text { if } n \geq 4 \text { is even; } \\ 5 \cdot 2^{\frac{n-5}{2}}, & \text { if } n \geq 5 \text { is odd. }\end{cases}
$$

Furthermore, $\operatorname{mi}(G)=g^{\prime}(n, 1)$ if and only if $G \in G^{\prime}(n, 1)$, where

$$
G^{\prime}(n, 1)=\left\{\begin{array}{l}
P_{4} \cup \frac{n-4}{2} P_{2}, C_{3} \cup T(2 s-3) \cup \frac{n-2 s}{2} P_{2} \text { or } C(2 s, 1) \cup \frac{n-2 s}{2} P_{2}, \text { if } n \geq 4 \text { is even }(s \geq 2) ; \\
C_{5} \cup \frac{n-5}{2} P_{2} \text { or } C(5,1) \cup \frac{n-5}{2} P_{2}, \text { if } n \geq 5 \text { is odd. }
\end{array}\right.
$$

Theorem 5. ([9]) If $H$ is a connected graph of order $n \geq 3$ with at most one cycle, then $\operatorname{mi}(H) \leq h(n, 1)$, where

$$
h(n, 1)= \begin{cases}2^{\frac{n-1}{2}}+1, & \text { if } n \geq 3 \text { is odd; } \\ 3 \cdot 2^{\frac{n-4}{2}}, & \text { if } n \geq 4 \text { is even. }\end{cases}
$$

Furthermore, $\operatorname{mi}(H)=h(n, 1)$ if and only if $H \in H(n, 1)$ (see Figure 2), where

$$
H(n, 1)= \begin{cases}K_{3} * \frac{n-3}{2} K_{2} \text { or } C_{5}, & \text { if } n \geq 3 \text { is odd; } \\ K_{1} *\left(K_{3} \cup \frac{n-4}{2} K_{2}\right) \text { or } P_{4}, & \text { if } n \geq 4 \text { is even. }\end{cases}
$$

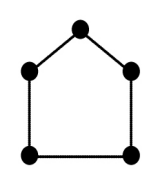

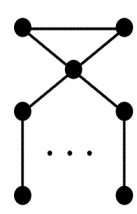

$n \geq 3$ odd

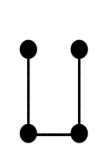

or

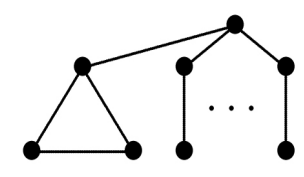

$n \geq 4$ even

Figure 2. The extremal graphs $H(n, 1)$ for $n \geq 3$. 


\section{The Alternative Proof}

In this section, we give an alternative proof to determine the largest number of maximal independent sets among all connected graphs of order $n \geq 12$, which contain at most two cycles. We also characterize the extremal graph achieving this maximum value.

Theorem 6. If $H$ is a connected graph of order $n \geq 12$ with at most two cycles, then $\operatorname{mi}(H) \leq h(n, 2)$, where

$$
h(n, 2)= \begin{cases}3 \cdot 2^{\frac{n-4}{2}}+2, & \text { if } n \geq 12 \text { is even; } \\ 9 \cdot 2^{\frac{n-7}{2}}, & \text { if } n \geq 13 \text { is odd. }\end{cases}
$$

Furthermore, $\operatorname{mi}(H)=h(n, 2)$ if and only if $H \in H(n, 2)$, where

$$
H(n, 2)= \begin{cases}K_{3} \times\left(K_{3} \cup \frac{n-6}{2} K_{2}\right), & \text { if } n \geq 12 \text { is even; } \\ K_{1} \times\left(2 K_{3} \cup \frac{n-7}{2} K_{2}\right), & \text { if } n \geq 13 \text { is odd. }\end{cases}
$$

A unicyclic graph is a connected graph having one cycle. The order of a unicyclic graph is at least three. The following lemmas will be needed in the proof of main theorem.

Lemma 4. Suppose that $G=T \cup H$ is the union of a tree $T$ and a unicyclic graph $H$, where $|G|=n \geq 6$. Then $\operatorname{mi}(G) \leq 2 \cdot h(n-2,1)$. The equality holds if and only if $G=K_{2} \cup H(n-2,1)$ or $G=K_{3} \cup B\left(1, \frac{n-4}{2}\right)$.

Proof. Let $|T|=k$. Note that $H$ has one cycle, then $|H|=n-k \geq 3$. We consider two cases.

Case 1. $n \geq 6$ is even.

By Lemma 2, Theorem 1 and Theorem 5, we have

$$
\begin{aligned}
& m i(G)=m i(T) \cdot m i(H) \leq t(k) \cdot h(n-k, 1) \\
& = \begin{cases}2^{\frac{k-1}{2}} \cdot\left(2^{\frac{n-k-1}{2}}+1\right), & \text { if } 1 \leq k \leq n-3 \text { is odd; } \\
\left(2^{\frac{k-2}{2}}+1\right) \cdot\left(3 \cdot 2^{\frac{n-k-4}{2}}\right), & \text { if } 2 \leq k \leq n-4 \text { is even; }\end{cases} \\
& = \begin{cases}2^{\frac{n-2}{2}}+2^{\frac{k-1}{2}}, & \text { if } 1 \leq k \leq n-3 \text { is odd; } \\
3\left(2^{\frac{n-6}{2}}+2^{\frac{n-k-4}{2}}\right), & \text { if } 2 \leq k \leq n-4 \text { is even; }\end{cases} \\
& \leq \begin{cases}2^{\frac{n-2}{2}}+2^{\frac{(n-3)-1}{2}}, & \text { if } 1 \leq k \leq n-3 \text { is odd; } \\
3\left(2^{\frac{n-6}{2}}+2^{\frac{n-(2)-4}{2}}\right), & \text { if } 2 \leq k \leq n-4 \text { is even; }\end{cases} \\
& =\left\{\begin{array}{ll}
3 \cdot 2^{\frac{n-4}{2}}, & \text { if } 1 \leq k \leq n-3 \text { is odd; } \\
3 \cdot 2^{\frac{n-4}{2}}, & \text { if } 2 \leq k \leq n-4 \text { is even; }
\end{array}= \begin{cases}t(n-3) \cdot 3, & \text { if } 1 \leq k \leq n-3 \text { is odd; } \\
2 \cdot h(n-2,1), & \text { if } 2 \leq k \leq n-4 \text { is even; }\end{cases} \right. \\
& =2 \cdot h(n-2,1)
\end{aligned}
$$


If the equality holds, then $\operatorname{mi}(G)=t(n-3) \cdot h(3,1)$ or $m i(G)=t(2) \cdot h(n-2,1)$. Hence the equality holds if and only if $G=K_{2} \cup H(n-2,1)$ or $G=K_{3} \cup B\left(1, \frac{n-4}{2}\right)$.

Case 2. $n \geq 7$ is odd.

By Lemma 3 and since $n \geq 7$, $2^{\frac{k-2}{2}}+2^{\frac{n-k-1}{2}} \leq \max \left\{2^{\frac{(2)-2}{2}}+2^{\frac{n-(2)-1}{2}}, 2^{\frac{(n-3)-2}{2}}+2^{\frac{n-(n-3)-1}{2}}\right\}=2^{\frac{n-3}{2}}+1$ for $2 \leq k \leq n-3$.

By Theorem 1 and Theorem 5, we have

$$
\begin{aligned}
& m i(G)=m i(T) \cdot m i(H) \leq t(k) \cdot h(n-k, 1) \\
& = \begin{cases}2^{\frac{k-1}{2}} \cdot\left(3 \cdot 2^{\frac{n-k-4}{2}}\right), & \text { if } 1 \leq k \leq n-4 \text { is odd; } \\
\left(2^{\frac{k-2}{2}}+1\right) \cdot\left(2^{\frac{n-k-1}{2}}+1\right), & \text { if } 2 \leq k \leq n-3 \text { is even; }\end{cases} \\
& = \begin{cases}3 \cdot 2^{\frac{n-5}{2}}, & \text { if } 1 \leq k \leq n-4 \text { is odd; } \\
2^{\frac{n-3}{2}}+2^{\frac{k-2}{2}}+2^{\frac{n-k-1}{2}}+1, & \text { if } 2 \leq k \leq n-3 \text { is even; }\end{cases} \\
& \leq \begin{cases}3 \cdot 2^{\frac{n-5}{2}}, & \text { if } 1 \leq k \leq n-4 \text { is odd; } \\
2^{\frac{n-3}{2}}+1+\left(2^{\frac{n-3}{2}}+1\right), & \text { if } 2 \leq k \leq n-3 \text { is even; }\end{cases} \\
& \leq 2^{\frac{n-1}{2}}+2=2 \cdot h(n-2,1) \text {. }
\end{aligned}
$$

If the equality holds, then $\operatorname{mi}(G)=t(2) \cdot h(n-2,1)$. Hence the equality holds if and only if $G=K_{2} \cup H(n-2,1)$.

By case 1 and case 2 , we have that $\operatorname{mi}(G) \leq 2 \cdot h(n-2,1)$. The equality holds if and only if $G=K_{2} \cup H(n-2,1)$ or $G=K_{3} \cup B\left(1, \frac{n-4}{2}\right)$.

Lemma 5. Suppose that $F$ is a forest of order $n \geq 4$ having at most two components. Then $\mathrm{mi}(F) \leq 2 \cdot t(n-2)$ and the equality holds if and only if $F=K_{2} \cup T(n-2)$ or $F=B\left(1, \frac{n-1}{2}\right)$.

Proof. Let $F$ be a forest of order $n \geq 4$ having at most two components such that $m i(F)$ as large as possible. Then $m i(F) \geq m i\left(K_{2} \cup T(n-2)\right)=2 \cdot t(n-2)$. If $F$ has one component, then $F$ is a tree and, by Theorem $1,2 \cdot t(n-2) \leq m i(F) \leq t(n)$. Then $n$ is odd and $\operatorname{mi}(F)=t(n)$. By Theorem $1, F=B\left(1, \frac{n-1}{2}\right)$. Now we assume that $F$ have two components. Let $T_{1}$ and $T_{2}$ be the components of $F$, where $\left|T_{1}\right|=s$. We consider two cases.

Case 1. $n \geq 4$ is even.

By Lemma 3, $2^{\frac{s-2}{2}}+2^{\frac{n-s-2}{2}} \leq \max \left\{2^{\frac{(2)-2}{2}}+2^{\frac{n-(2)-2}{2}}, 2^{\frac{(n-2)-2}{2}}+2^{\frac{n-(n-2)-2}{2}}\right\}=2^{\frac{n-4}{2}}+1$ for 
$2 \leq s \leq n-2$. By Lemma 2 and Theorem 1 , then

$$
\begin{aligned}
& 2 \cdot t(n-2)=2\left(2^{\frac{n-4}{2}}+1\right) \leq m i(F)=m i\left(T_{1}\right) \cdot m i\left(T_{2}\right) \leq t(s) \cdot t(n-s) \\
& = \begin{cases}2^{\frac{s-1}{2}} \cdot\left(2^{\frac{n-s-1}{2}}\right), & \text { if } 1 \leq s \leq n-1 \text { is odd; } \\
\left(2^{\frac{s-2}{2}}+1\right) \cdot\left(2^{\frac{n-s-2}{2}}+1\right), & \text { if } 2 \leq s \leq n-2 \text { is even; }\end{cases} \\
& = \begin{cases}2^{\frac{n-2}{2}}, & \text { if } 1 \leq s \leq n-1 \text { is odd; } \\
2^{\frac{n-4}{2}}+1+\left(2^{\frac{s-2}{2}}+2^{\frac{n-s-2}{2}}\right), & \text { if } 2 \leq s \leq n-2 \text { is even; }\end{cases} \\
& \leq \begin{cases}2^{\frac{n-2}{2}}, & \text { if } 1 \leq s \leq n-1 \text { is odd; } \\
2^{\frac{n-4}{2}}+1+\left(2^{\frac{n-4}{2}}+1\right), & \text { if } 2 \leq s \leq n-2 \text { is even; }\end{cases} \\
& = \begin{cases}2^{\frac{n-2}{2}}, & \text { if } 1 \leq s \leq n-1 \text { is odd; } \\
2^{\frac{n-2}{2}}+2, & \text { if } 2 \leq s \leq n-2 \text { is even; }\end{cases} \\
& \leq 2\left(2^{\frac{n-4}{2}}+1\right)=2 \cdot t(n-2) \text {. }
\end{aligned}
$$

The equalities hold and $m i(F)=t(2) \cdot t(n-2)$. By Theorem 1, $F=K_{2} \cup T(n-2)$. The equality holds if and only if $F=K_{2} \cup T(n-2)$.

Case 2. $n \geq 5$ is odd.

Then $F$ has exactly one even component, we assume that $s \geq 2$ is even. By Theorem 1 , then

$$
\begin{aligned}
& 2 \cdot t(n-2)=2^{\frac{n-1}{2}} \leq m i(F)=m i\left(T_{1}\right) \cdot m i\left(T_{2}\right) \leq t(s) \cdot t(n-s)=\left(2^{\frac{s-2}{2}}+1\right) \cdot\left(2^{\frac{n-s-1}{2}}\right) \text {. The } \\
& =2^{\frac{n-3}{2}}+2^{\frac{n-s-1}{2}} \leq 2^{\frac{n-3}{2}}+2^{\frac{n-(2)-1}{2}}=2^{\frac{n-1}{2}}=2 \cdot t(n-2)
\end{aligned}
$$

equalities hold and $m i(F)=t(2) \cdot t(n-2)$. The equality holds if and only if $F=K_{2} \cup T(n-2)$.

The following is the proof of Theorem 6 .

Proof. Let $H$ be a connected graph of order $n \geq 12$ with at most two cycles such that $m i(H)$ as large as possible. Then $m i(H) \geq m i(H(n, 2))=h(n, 2)$. Since $\operatorname{mi}(H) \geq h(n, 2)>h(n, 1)$, by Theorem $5, H$ have at least two cycles. That means that $H$ have exactly two cycles and $H \neq C_{n}$. Let $v$ be a vertex lying on some cycle such that $\operatorname{deg}(v)$ is as large as possible. Since $H \neq C_{n}$, we can see that $\operatorname{deg}(v)=s \geq 3$. The graph $H-v$ is a graph of order $n-1$ with at most one cycle. We consider two cases.

Case 1. $H-v=G(n-1,1)$.

Then $H-v=K_{3} \cup \frac{n-4}{2} P_{2}$ or $H-v=\frac{n-1}{2} P_{2}$. Since $H$ is connected, this means 
that $v$ connects to every component of $G(n-1,1)$. Then $m i(H-N[v])$ has at most one edge, then $\operatorname{mi}(H-N[v]) \leq 2$. So we have

$$
\begin{aligned}
h(n, 2) & \leq m i(H) \leq m i(H-v)+m i(H-N[v]) \leq g(n-1,1)+2 \\
& = \begin{cases}3 \cdot 2^{\frac{n-4}{2}}+2, & \text { if } n \geq 12 \text { is even; } \\
2^{\frac{n-1}{2}}+2, & \text { if } n \geq 13 \text { is odd; }\end{cases}
\end{aligned}
$$

Then $\operatorname{mi}(G-N[v])=2$ and $n \geq 12$ is even. So $H-v=K_{3} \cup \frac{n-4}{2} P_{2}$. Note that $H$ has two cycles, hence $H=K_{3} \times\left(K_{3} \cup \frac{n-6}{2} K_{2}\right)=H(n, 2)$ for even $n \geq 12$.

Case 2. $H-v \neq G(n-1,1)$.

Let $\operatorname{deg}(v)=d$. Then the subgraph $H-N[v]$ is a graph of order $n-d-1$ having at most one cycle. By Theorem 3, $m i(H-N[v]) \leq g(n-d-1)$. By Theorem 4, $m i(H-v) \leq g^{\prime}(n-1,1)$. By Lemma 1 and Theorem 4, then

$$
\begin{aligned}
g(n-d-1) \geq & m i(H-N[v]) \geq m i(H)-m i(H-v) \geq h(n, 2)-g^{\prime}(n-1,1) \\
& = \begin{cases}\left(3 \cdot 2^{\frac{n-4}{2}}+2\right)-\left(5 \cdot 2^{\frac{n-6}{2}}\right), & \text { if } n \geq 12 \text { is even; } \\
\left(9 \cdot 2^{\frac{n-7}{2}}\right)-\left(3 \cdot 2^{\frac{n-5}{2}}\right), & \text { if } n \geq 13 \text { is odd; }\end{cases} \\
= & \left\{\begin{array}{ll}
2^{\frac{n-6}{2}}+2, & \text { if } n \geq 12 \text { is even; } \\
3 \cdot 2^{\frac{n-7}{2}}, & \text { if } n \geq 13 \text { is odd; }
\end{array}= \begin{cases}g(n-6,1)+2, & \text { if } n \geq 12 \text { is even; } \\
g(n-4,1), & \text { if } n \geq 13 \text { is odd; }\end{cases} \right.
\end{aligned}
$$

Then

$$
\operatorname{deg}(v)=d \leq \begin{cases}4, & \text { if } n \geq 12 \text { is even; } \\ 3, & \text { if } n \geq 13 \text { is odd }\end{cases}
$$

Claim. $\operatorname{deg}(v)=3$.

Suppose that $\operatorname{deg}(v)=4$, then $n$ is even. By Theorem 3, mi $(H-N[v]) \leq g(n-5,1)$ and

$$
\begin{aligned}
& m i(H-v) \geq m i(H)-m i(H-N[v]) \geq h(n, 2)-g(n-5,1)=\left(3 \cdot 2^{\frac{n-4}{2}}+2\right)-3 \cdot 2^{\frac{n-8}{2}} . \\
& =9 \cdot 2^{\frac{n-8}{2}}+2>2^{\frac{n-2}{2}}=f(n-1)
\end{aligned}
$$

By Theorem 2, $H-v$ is not a forest and $H-v$ has one cycle. Let $H^{\prime \prime}$ be the component of $H-v$ having one cycle and $F=H-v-V\left(H^{\prime \prime}\right)$, where $\left|H^{\prime \prime}\right|=s \geq 3$. Note that $H$ has two cycles and $v$ is lying on some cycle. Thus $v$ has two edges incident to some component of $H-v$. Since $\operatorname{deg}(v)=4$, the number of the components of $H-v$ is at most three. Thus $F$ is either a tree or the union of two trees. By Lemma 5, $m i(F) \leq 2 \cdot t(n-s-3)$. By Lemma 3,

$2^{\frac{s+1}{2}}+2^{\frac{n-s-3}{2}} \leq \max \left\{2^{\frac{(3)+1}{2}}+2^{\frac{n-(3)-3}{2}}, 2^{\frac{(n-5)+1}{2}}+2^{\frac{n-(n-5)-3}{2}}\right\} \leq 2^{\frac{n-4}{2}}+2$ for $3 \leq s \leq n-5$. By 
Theorem 5, $m i\left(H^{\prime \prime}\right) \leq h(s, 1)$. Note that $m i(H-v) \geq 9 \cdot 2^{\frac{n-8}{2}}+2$. By Lemma 2 and Lemma 5,

$$
\begin{aligned}
9 \cdot 2^{\frac{n-8}{2}}+2 \leq & (H-v)=m i(F) \cdot m i\left(H^{\prime \prime}\right) \leq \begin{cases}2 \cdot h(n-3,1), & \text { if } s=n-3 ; \\
2 \cdot t(n-s-3)) \cdot h(s, 1), & \text { if } s \neq n-3 ;\end{cases} \\
& = \begin{cases}2 \cdot\left(2^{\frac{n-4}{2}}+1\right), & \text { if } s=n-3 ; \\
\left.2 \cdot\left(2^{\frac{n-s-5}{2}}+1\right)\right) \cdot\left(2^{\frac{s-1}{2}}+1\right), & \text { if } 3 \leq s \leq n-5 \text { is odd; } \\
\left(2 \cdot 2^{\frac{n-s-4}{2}}\right) \cdot\left(3 \cdot 2^{\frac{s-4}{2}}\right), & \text { if } 2 \leq s \leq n-4 \text { is even; }\end{cases} \\
& = \begin{cases}2^{\frac{n-2}{2}}+2, & \text { if } s=n-3 ; \\
2^{\frac{n-4}{2}}+2+\left(2^{\frac{s+1}{2}}+2^{\frac{n-s-3}{2}}\right), & \text { if } 3 \leq s \leq n-5 \text { is odd; } \\
3 \cdot 2^{\frac{n-6}{2}}, & \text { if } 2 \leq s \leq n-4 \text { is even; }\end{cases} \\
& \leq \begin{cases}2^{\frac{n-2}{2}}+2, & \text { if } s=n-3 ; \\
2^{\frac{n-4}{2}}+2+\left(2^{\frac{n-4}{2}}+2\right), & \text { if } 3 \leq s \leq n-5 \text { is odd; } \leq 2^{\frac{n-2}{2}}+4<9 \cdot 2^{\frac{n-8}{2}}+2, \\
3 \cdot 2^{\frac{n-6}{2}}, & \text { if } 2 \leq n-4 \text { is even; }\end{cases}
\end{aligned}
$$

where $n \geq 12$. This is a contradiction, so $\operatorname{deg}(v)=3$.

By Claim, $\operatorname{deg}(v)=3$. Note that $H$ has two cycles and $v$ is lying on some cycle. Thus $v$ has two edges incident to some component of $H-v$. Since $\operatorname{deg}(v)=3$, the number of the components of $H-v$ is at most two. Thus $H-v=T \cup H^{\prime}$, where $T$ is a tree and $H^{\prime}$ is a unicyclic graph. By Lemma 4 and Theorem 3, then

$$
\begin{aligned}
& m i(H-v)=m i(T) \cdot m i\left(H^{\prime}\right) \leq 2 \cdot h(n-3,1)=2 \cdot h(n-3,1) \text { and } \\
& \operatorname{mi}(H-N[v]) \leq g(n-4,1) \text {. So } \\
& h(n, 2) \leq m i(H) \leq m i(H-v)+m i(H-N[v]) \\
& \leq 2 \cdot h(n-3,1)+g(n-4,1) \\
& = \begin{cases}2\left(2^{\frac{n-4}{2}}+1\right)+2^{\frac{n-4}{2}}, & \text { if } n \geq 12 \text { is even; } \\
2\left(3 \cdot 2^{\frac{n-7}{2}}\right)+3 \cdot 2^{\frac{n-7}{2}}, & \text { if } n \geq 13 \text { is odd; }\end{cases} \\
& = \begin{cases}3 \cdot 2^{\frac{n-4}{2}}+2, & \text { if } n \geq 12 \text { is even; } \\
9 \cdot 2^{\frac{n-7}{2}}, & \text { if } n \geq 13 \text { is odd. }\end{cases} \\
& =h(n, 2) \text {. }
\end{aligned}
$$


The equalities hold. Then $H-N[v]=G(n-4,1)$ and, by Lemma 4,

$H-v=K_{2} \cup H(n-3,1)$ or $K_{3} \cup B\left(1, \frac{n-5}{2}\right)$. If $H-v=K_{3} \cup B\left(1, \frac{n-5}{2}\right)$, then $n$ is

odd and $H-N[v] \neq K_{3} \cup \frac{n-7}{2} K_{2}$. That is $H-N[v] \neq G(n-4,1)$, this is a contradiction. Thus $H-v=K_{2} \cup H(n-3,1)$. If $n$ is even, where $n \geq 12$, then

$H-v=K_{2} \cup H(n-3,1)=K_{2} \cup\left(K_{3} \times \cup \frac{n-6}{2} K_{2}\right)$ and

$H-N[v]=G(n-4,1)=\frac{n-4}{2} K_{2}$. Then, there exists a vertex $u \in V(H-v)$ lying on some cycle such that $\operatorname{deg}(u) \geq \frac{n}{2}>3$. This contradicts to the claim, so $n$ is odd. Thus $H-v=K_{2} \cup H(n-3,1)=K_{2} \cup\left(K_{1} *\left(K_{3} \cup \frac{n-7}{2} K_{2}\right)\right)$ and

$H-N[v]=G(n-4,1)=K_{3} \cup \frac{n-7}{2} K_{2}$. Hence $H=K_{1} \times\left(2 K_{3} \cup \frac{n-7}{2} K_{2}\right)=H(n, 2)$

for odd $n \geq 13$.

\section{Acknowledgements}

The authors are grateful to the referees for the helpful comments.

\section{References}

[1] Ying, G.C., Meng, Y.Y., Sagan, B.E. and Vatter, V.R. (2006) Maximal Independent Sets in Graphs with at Most $r$ Cycles. Journal of Graph Theory, 53, 270-282.

http://dx.doi.org/10.1002/jgt.20185

[2] Moon, J.W. and Moser, L. (1965) On Cliques in Graphs. Israel Journal of Mathematics, 3, 23-28. http://dx.doi.org/10.1007/BF02760024

[3] Jou, M.J. and Chang, G.J. (1995) Survey on Conunting Maximal Independent Sets. In: Tangmance, S. and Schulz, E., Eds., Proceedings of the Second Asian Mathematical Conference, World Scientific, Singapore City, 265-275.

[4] Jin, Z. and Li, X. (2008) Graphs with the Second Largest Number of Maximal Independent Sets. Discrete Mathematics, 308, 5864-5870. http://dx.doi.org/10.1016/j.disc.2007.10.032

[5] Prodinger, H. and Tichy, R.F. (1982) Fibonacci Numbers of Graphs. The Fibonacci Quarterly, 20, 16-21.

[6] Knopfmacher, A., Tichy, R.F., Wagner, S. and Ziegler, V. (2007) Graphs, Partitions and Fibonacci Numbers. Discrete Applied Mathematics, 155, 1175-1187.

http://dx.doi.org/10.1016/j.dam.2006.10.010

[7] Wagner, S.G. (2006) The Fibonacci Number of Generalized Petersen Graphs. The Fibonacci Quarterly, 44, 362-367.

[8] Jou, M.J. and Chang, G.J. (2002) Algorithmic Aspects of Counting Independent Sets. Ars Combinatoria, 65, 265-277.

[9] Jou, M.J. and Chang, G.J. (1997) Maximal Independent Sets in Graphs with at Most One Cycle. Discrete Applied Mathematics, 79, 67-73. http://dx.doi.org/10.1016/S0166-218X(97)00033-4

[10] Sagan, B.E. and Vatter, V.R. (2006) Maximal and Maximum Independent Sets in Graphs 
with at Most $r$ Cycles. Journal of Graph Theory, 53, 283-314.

http://dx.doi.org/10.1002/jgt.20186

[11] Jou, M.J. (2012) The Second Largest Number of Maximal Independent Sets in Connected Graphs with at Most One Cycle. Journal of Combinatorial Optimization, 24, 192-201. http://dx.doi.org/10.1007/s10878-011-9376-4

[12] Hujter, M. and Tuza, Z. (1993) The Number of Maximal Independent Sets in Triangle-Free Graphs. SIAM: SIAM Journal on Discrete Mathematics, 6, 284-288.

http://dx.doi.org/10.1137/0406022

[13] Jou, M.J. (1991) The Number of Maximal Independent Sets in Graphs. Master Thesis, Department of Mathematics, National Central University, Taoyuan.

Submit or recommend next manuscript to SCIRP and we will provide best service for you:

Accepting pre-submission inquiries through Email, Facebook, LinkedIn, Twitter, etc. A wide selection of journals (inclusive of 9 subjects, more than 200 journals)

Providing 24-hour high-quality service

User-friendly online submission system

Fair and swift peer-review system

Efficient typesetting and proofreading procedure

Display of the result of downloads and visits, as well as the number of cited articles Maximum dissemination of your research work

Submit your manuscript at: http://papersubmission.scirp.org/ 